\title{
BMJ Open Resilience and quality of life (QoL) of head and neck cancer and brain tumour survivors in Pakistan: an analytical cross-sectional study protocol
}

\author{
Nida Zahid, ${ }^{\oplus 1}$ Wardah Khalid, ${ }^{1}$ Khabir Ahmad, ${ }^{1}$ Shireen Shehzad Bhamani, ${ }^{2}$ \\ lqbal Azam, ${ }^{3}$ Nargis Asad, ${ }^{4}$ Adnan Abdul Jabbar, ${ }^{5}$ Mumtaz Khan, ${ }^{1}$ Ather Enam ${ }^{1}$
}

To cite: Zahid N, Khalid W, Ahmad K, et al. Resilience and quality of life $(\mathrm{Q} \mathrm{L})$ of head and neck cancer and brain tumour survivors in Pakistan: an analytical cross-sectional study protocol. BMJ Open 2019;9:e029084. doi:10.1136/ bmjopen-2019-029084

- Prepublication history for this paper is available online. To view these files, please visit the journal online (http://dx.doi. org/10.1136/bmjopen-2019029084).

Received 14 January 2019 Revised 09 August 2019 Accepted 23 August 2019

\section{Check for updates}

(c) Author(s) (or their employer(s)) 2019. Re-use permitted under CC BY-NC. No commercial re-use. See rights and permissions. Published by BMJ.

${ }^{1}$ Department of Surgery, Aga Khan University Hospital, Karachi, Pakistan

${ }^{2}$ School of Nursing and Midwifery, Aga Khan University Hospital, Karachi, Pakistan ${ }^{3}$ Department of Community Health Sciences, Aga Khan University Hospital, Karachi, Pakistan

${ }^{4}$ Department of Psychiatry, Aga Khan University Hospital, Karachi, Pakistan

${ }^{5}$ Department of Oncology, Aga Khan University Hospital, Karachi, Pakistan

Correspondence to

Dr Nida Zahid;

nida.zahid@aku.edu

\section{ABSTRACT}

Background Cancer is a devastating disease and has detrimental effects on the quality of life (QoL) of cancer survivors and interferes with their treatment compliance. The aim of the study is to assess resilience and QoL among cancer survivors and to evaluate the important factors affecting their resilience and $\mathrm{Q} o \mathrm{~L}$, with respect to the Pakistani cultural context.

Method and analysis A cross-sectional study will be conducted at a tertiary care hospital in Karachi, Pakistan. A minimum sample size of 250 head and neck cancers and 250 brain tumour survivors with $10 \%$ inflation for non-response rate will be required. The SD of $Q 0 L$ and resilience will range from 16.5 to 40.8 for head and neck cancer, and 12.7 to 34.1 for brain tumour, at $5 \%$ level of significance, with 2.5 precision. QoL will be assessed by European Organization for Research and Treatment of Cancer (EORTC) QLQ-C30, EORTC QLQ-H\&N35 and EORTC QLQ-BN20 and resilience will be evaluated by Wagnild and Young's 14-item scale. Mean \pm SD will be reported for resilience and QoL scores. Unadjusted and adjusted $\beta$-coefficients, with $95 \% \mathrm{Cl}$, will be reported by using multiple linear regression analysis. Correlation analysis will also be performed using Pearson or Spearman rank correlation coefficients. A p value of $<0.05$ will be considered significant.

Ethics and dissemination Ethical approval has been obtained from the Aga Khan University Pakistan's Ethical Review Committee. Written informed consent will be taken from the participants by trained research assistants. A trained psychologist will provide on-spot counselling to the participants and those identified with severe depression will be referred to a psychiatrist. The study materials will be kept under lock and key and the electronic data base will be password protected and will only be accessed by the research team. The study findings will be disseminated through publications conferences and workshops and research briefs.

Trial registration number Clinicaltrials.gov registry (NCT03466762).

\section{BACKGROUND}

Globally, cancer is the second leading cause of death. ${ }^{1}$ Approximately $70 \%$ of the deaths from cancer occur in the lower middle
Strengths and limitations of this study

Validated measures will be used for evaluating the outcomes and the independent variables.

- The overall quality of the study will be maintained through random spot-checks.

- The study results can be generalised to all cancer patients presenting at private tertiary care hospitals in Pakistan.

- To compare the quality of life and resilience within the two groups of patients, subgroup analysis will be performed by stratification.

income countries. Head and neck cancers are the sixth most common cancers worldwide, with $\sim 630000$ new cases diagnosed annually, causing 350000 deaths. ${ }^{23}$ Globally, brain tumours are also a significant source of cancer-related morbidity and mortality, with an overall incidence of $4-5 / 100000$ cases annually, contributing to $2 \%$ of all cancer deaths. ${ }^{4}$ And in Pakistan $~ 150000$ new cases of cancer are diagnosed annually, causing $60 \%-80 \%$ deaths. $^{5}$

Conventionally, the endpoints of medical care for cancer patients are focused on survival rate, local control rate or complication rate. ${ }^{6}$ These assessments do not capture the patients' mental and emotional wellbeing, ${ }^{6}$ although the diagnosis of cancer considerably affects a patient's emotional and psychological status ${ }^{7}$

Cancer patients suffer clinically important symptoms of emotional distress such as depression and anxiety ${ }^{8}$ that reduces their quality of life (QoL) and resilience and interferes with their treatment compliance..$^{9} 10$ Studies have found that cancer patients with similar diseases and treatment status have significantly different QoLs. ${ }^{11} 12$ It is believed that resilience is the main factor that causes 
patients with similar situations to have different perceptions about their QoL. ${ }^{13} 14$

Resilience is an important trait that contributes to a person's mental and physical well-being. Evidence suggests that resilience is related to motivation. This motivation to recover from physical or psychological traumatic events ${ }^{1516}$ minimises the impact of risk factors, thus increasing a person's ability to deal with challenges of life. ${ }^{17}$ Resilience, thus protects against psychosocial health-related issues, such as depression, anxiety, fear and helplessness, and helps to reduce their associated negative effects. ${ }^{18}$

Resilience has an important impact on the QoL of a cancer patient. Hence, over the last few years, QoL has become an important health-related outcome measure with regard to communities and healthcare systems. This outcome measure is based on multidimensional concept that incorporates: the subjective perceptions of positive and negative aspects of cancer symptoms, physical, emotional, social,cognitive functions, the disease symptoms and the side effects of treatment. ${ }^{719}$

There are several positive and negative factors that can influence a cancer patient's resilience and QoL. These are: illness-related risk, which include perceived illness, ambiguity and complexity, stress of symptoms, severity of illness; family protective factors, which include perceived social support from family and socioeconomic variables; social protective factors, which include perceived social support from friends, influence of others with similar conditions and perceived support from providers; individual risk factors including evasive, emotive and fatalistic coping measures/strategies; individual protective factors,

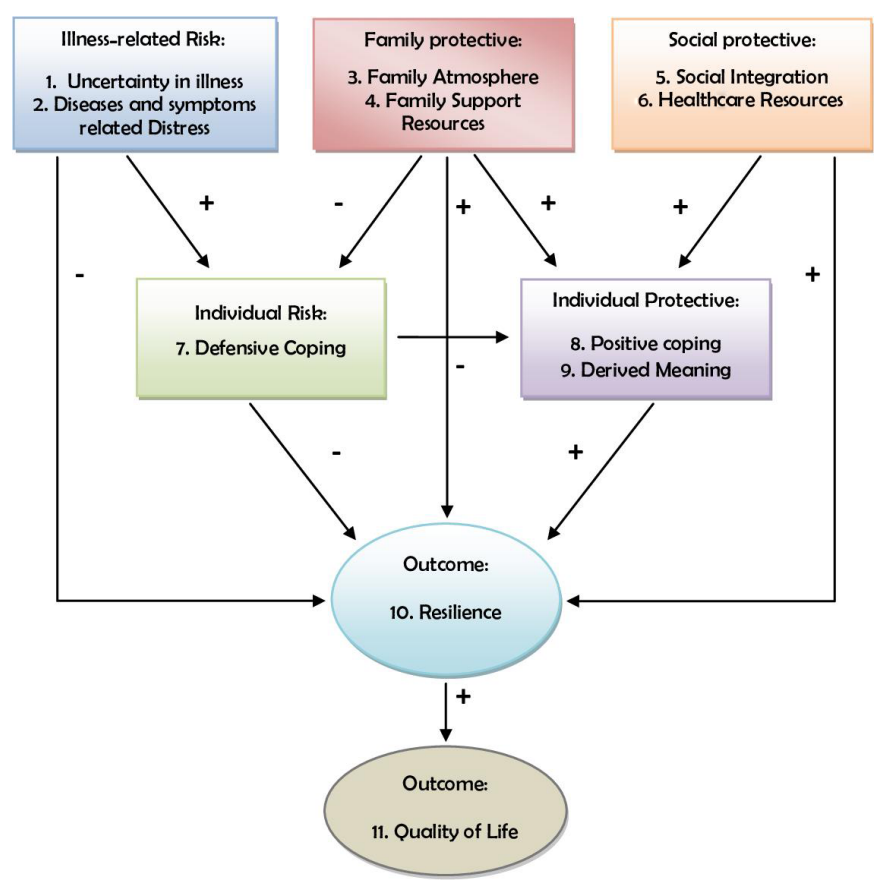

Figure 1 Conceptual framework of resilience and quality of life for cancer survivors adapted from 'the adolescent resilience model'. ${ }^{47}$ which include confrontive, optimistic and supportant coping, along with hope and spiritual factors (figure 1).

Studies have examined the influence of psychological resilience among cancer patients. ${ }^{20}{ }^{21}$ These studies from different parts of the world suggest that resilience is a protective factor against distress among cancer survivors $^{22-26}$ which indicates that cancer patients with high resilience require less psychosocial support to manage their stressful conditions, as compared with those with low resilience. ${ }^{20}$ One study reports that resilience mediates between cancer symptoms and distress and QoL among cancer survivors. Hence, resilience plays an important role in protecting them against the adverse effects of cancer symptoms. ${ }^{22}$ A systematic review of 24 studies on head and neck cancer patients reports that distress-related variables (depression, anxiety and distress) have a negative association with QoL outcomes. ${ }^{27}$

Moreover, resilience is a critical component for QoL at all stages; during diagnosis, treatment, survivorship and palliative care. It is an important trait for promoting positive psychosocial well-being. Early identification of psychological factors associated with post-treatment QoL is essential among those at increased risk of poorer outcomes, as this can aid in the development of interventions to improve their QoL. ${ }^{27}$

Limited evidence is available from the Pakistani context regarding resilience and QoL among cancer patients. To the best of the researchers' knowledge, this will be the first in-depth study to evaluate resilience and QoL among head and neck cancer and brain tumour patients in Pakistan. Resilience and QoL among them changes over time and may be modifiable towards increased well-being. This study will, therefore, enable designing of interventions in the future to improve resilience and QoL. In the light of literature, the objectives of this study are:

1. To determine the resilience and the QoL scores for head and neck cancer and brain tumour patients, at least 4 weeks post-treatment.

2. To evaluate important factors associated with resilience and QoL among head and neck cancer and brain tumour patients, at least 4 weeks post-treatment.

3. To examine the relationship between resilience and the QoL for head and neck cancer and brain tumour patients, at least 4 weeks post-treatment.

\section{METHODS}

\section{Study design}

To evaluate resilience and QoL among head and neck cancer and brain tumour patients and their associated factors an analytical cross-sectional study will be conducted. Resilience and QoL will be measured at least 4 weeks post-treatment.

\section{Study setting}

The study will be conducted at the Aga Khan University Hospital (AKUH) which is a Joint Commission International Accredition (JCIA-accredited) hospital, in 
Karachi-Pakistan. Karachi is the largest metropolitan city of Pakistan, a home to all major ethnicities living in this country. Aga Khan University Hospital (AKUH) is one of the largest private tertiary care hospitals that cater to different ethnic and socioeconomic groups of population in Karachi. The participants will be recruited from the surgical/oncology clinics at AKU. It has a multidisciplinary team that provides comprehensive care to cancer patients. The proposed duration of data collection will be 4-6 months.

\section{Study participants}

Men and women aged 18 years and above, who have received treatment for brain tumour and head and neck cancer at AKUH, fulfilling the below eligibility criteria, will be recruited. According to recent data, the prevalence of head and neck cancer is escalating in Pakistan and limited information is available about their QoL brain tumour also is an understudied area in Pakistan and there is dearth of information regarding their QoL. To maintain internal validity, the participants will be studied based on assumptions pertaining to their respective group.

\section{Eligibility criteria}

\section{Inclusion criteria}

1. Individuals aged 18 years and above, who have received treatment at AKUH for head and neck cancer or brain tumour.

2. Cancer survivors living in Pakistan for at least 3 months.

3. Patients who will give consent to participate in the study.

\section{Exclusion criteria}

1. Known cases of any psychiatric illness leading to disability (eg, manic disorder, schizophrenia and so on) as confirmed by medical records, will be excluded from the study as they may be on medications that might distort the results.

2. Patients on antidepressants prescribed by a psychiatrist

3. Participants with physical comorbidities, stroke and renal failure, will be excluded because these are debilitating diseases that will distort the results. Patients with Cardiovascular Diseases (CVD) / heart disease, diabetes or Chronic Obstructive Pulmonary Disease (COPD) will not be excluded as every $4^{\text {th }}$ Pakistani suffers from cardiovascular risk factors. If patient with these conditions are excluded, the majority of the participants will be ineligible and the required sample size will not be achieved. However, these comorbid conditions will be adjusted during analysis.

\section{Sampling technique}

Purposive sampling technique will be used for selecting the participants. The target population, that is, brain tumour and head and neck cancer patients who have received cancer treatment, will be approached by trained research assistants. The research assistants will be informed about the possible study participants who will

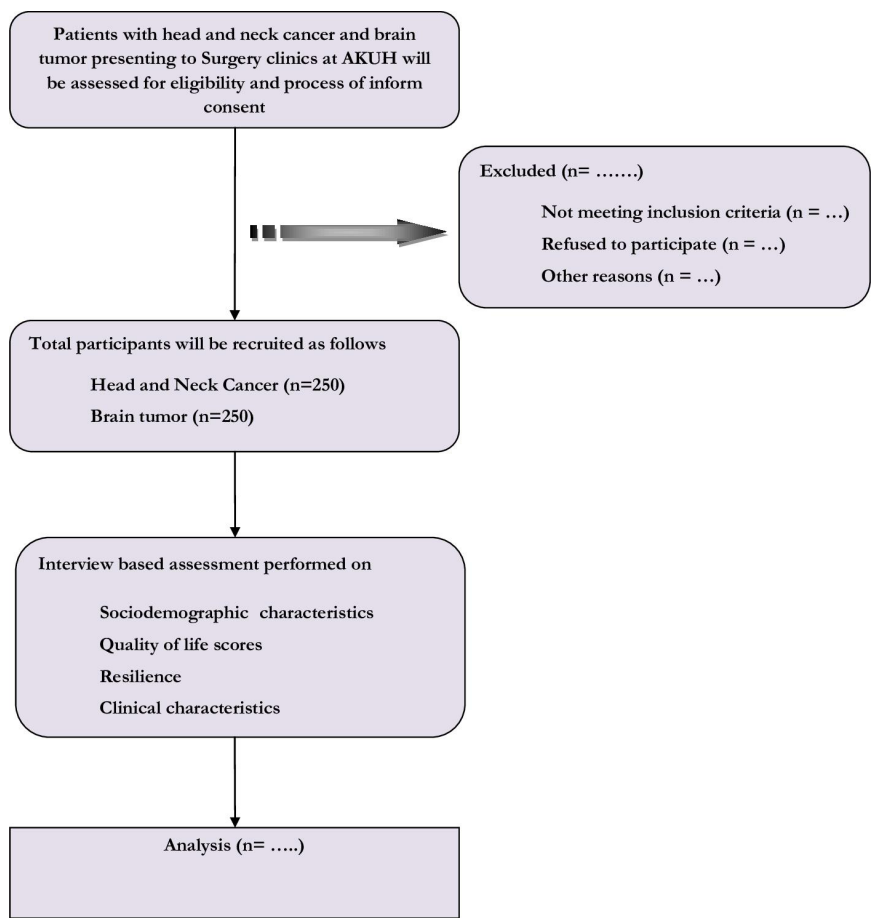

Figure 2 Participants recruitment plan flow diagram.

be coming in for their appointment, by the nurse. On the day of appointment, the participants will be screened for eligibility and if they fulfil the eligibility criteria and give consent to participate, they will be enrolled in the study (figure 2).

\section{Sample size calculation Head and neck cancer}

The sample size has been calculated based on mean QoL and resilience scores for head and neck cancer patients from previous studies. It has been calculated using one population mean formula, based on a SD range of 16.5$40.8,5 \%$ level of significance with precision of 2.5, and by adjusting the sample size for $10 \%$ non-response rate. The minimum sample size has been estimated to be $250 . .^{61428-31}$

\section{Brain tumour}

The sample size has been calculated based on previously reported estimates for QoL and resilience among patients with brain tumours. It has been calculated using one population mean formula, based on a SD range of 12.7-34.1, 5\% level of significance with precision of 2.5, and by adjusting the sample size for $10 \%$ non-response rate. The minimum sample size has been estimated to be $250 .^{32-35}$

\section{Assessment tools}

Resilience (Wagnild and Young's 14 items)

Resilience is the ability to rebound or spring back, the power of something to resume its original shape or position after compression or bending. ${ }^{36}$ Resilience is also defined as the "capacity of individuals exposed to a negative event, to maintain stability and healthy physical and 
psychological functioning. It is a defense mechanism, which permits people to grow in the face of adversity. ${ }^{, 37}$ The resilience tool that will be used has two versions; a long 25-item and short 14-item scale, using a 7-point rating likert scale. It comprises the five core characteristics of resilience, which include purposeful life, perseverance, equanimity, self-reliance and existential loneliness. ${ }^{38} \mathrm{~A}$ high score represents better resilience. The respondent's choice ranges from 1 (strongly disagree) to 7 (strongly agree). The scale uses total scores rather than scores of individual items. To measure resilience, the validated Urdu version of the resilience scale 14 (RS-14), which indicates moderate negative correlation of resilience with depression and anxiety $(\mathrm{r}=-0.31)$, and moderate positive significant correlation of resilience with life satisfaction $(\mathrm{r}=0.40)$ will be used. The test-retest correlation coefficients and Cronbach's alpha for RS-14 are 0.49 and 0.76, respectively. ${ }^{39}$

QoL (EORTC QLQ-C30, EORTC QLQ-H\&N35 and EORTC QLQ-BN20) QoL is defined by the WHO as 'Individual's perceptions of their position in life in the context of the culture and value systems and their goals, expectations, standards and concerns'. ${ }^{40}$ The QoL of the cancer survivors will be assessed by the European Organization for Research and Treatment of Cancer (EORTC) QLQ-C30 tool. ${ }^{41}$ It is composed of multi-item scales and single-item measures. These include five functional scales, three symptom scales, a global health status QoL scale and six single items. All the scales and single-item measures scores range from 0 to 100 . A high score on the scale represents a higher response level. Specific questionnaires will also be administered to evaluate the QoL of patients with brain tumours and head and neck cancer via EORTC QLQ-BN20 and EORTC QLQ-H\&N35, respectively. Since the tool has not been validated in Urdu, therefore, in this study content validation will be conducted through a panel of experts and the content validity index (CVI) will be calculated. The panel of experts will comprise head and neck surgeons, neurosurgeons, an oncologist, an epidemiologist, a biostatistician and a psychologist. They will be asked to provide their expert suggestions for improving the tool according to the Pakistani cultural context, in Urdu. Each and every expert will rate the tool regarding the relevancy and clarity of each question. The responses will be rated on a scale from not relevant to highly relevant. Based on expert scores, the CVI will be calculated. CVI quantifies the level of content validity by calculating the percentage agreement between experts. ${ }^{42}$ CVI of $>0.8$ indicates high level of agreement among the experts. ${ }^{43}$ Permission has been granted by the QoL tool developers for content validation.

\section{Sociodemography and clinical characteristics}

The information on demographic variables will be collected on aspects like; age, gender, ethnicity, education, family status, number of people actively working, monthly household income and employment status of the individuals. The socioeconomic determinant will include education, occupation and family income. Information on comorbid conditions such as hypertension, diabetes, cardiovascular disease, addiction history (including smoking and substance abuse) will also be evaluated. Data on important major recent life events, such as death of child, spouse or any other event that has affected their lives will also be collected. Clinical characteristics and management of brain tumour and head and neck cancer will also be assessed by taking information from the patients on tumour type, site of tumour, type of surgery, type of chemotherapy and/or radiotherapy.

\section{Psychosocial characteristics}

The participants' depression and anxiety will be assessed using the Hospital Anxiety and Depression Scale (HADS),${ }^{44}$ and social support will be determined via the Enriched Social Support Instrument (ESSI). ${ }^{9}$

\section{Hospital anxiety and depression (HADS)}

The Hospital Anxiety and Depression Scale (HADS) will be administered to assess depression and anxiety among the participants. This tool was developed to assess depression, anxiety and emotional distress among patients who were treated for a variety of clinical problems. HADS encompasses 14 items, equally subdivided into two scales, one measuring anxiety and the other depression. For instance, the item 'Worrying thoughts go through my mind' assesses anxiety, whereas the item 'I have lost interest in my appearance' evaluates depression. All the responses are on an ordinal four-point scale. ${ }^{44}$ To measure anxiety and depression, the Urdu version of HADS will be used. $^{45}$

\section{Social support by ESSI}

Social support can ease the coping process, or help people overcome or adapt to a stressful event. The ESSI is a 7-item scale that primarily measures functional social support and emotional support. A total score of 18 or less on items 1, 2, 3, 5 and 6 is considered as low social support. ${ }^{9}$ To assess social support, the validated Urdu version of ESSI, with a CVI for relevance, and clarity of 0.95 and 0.97 , respectively, and Cronbach's alpha $0.82^{46}$ will be used.

\section{Explanatory questions to evaluate culturally relevant theme}

Lastly, an explanatory questionnaire will be administered to examine the factors that have affected the lives of the cancer patients and also to examine the different coping tactics used by the patients and their families to combat this disease.

\section{Statistical analysis}

Analysis will be performed using the STATA V.12. Descriptive statistics will be computed for categorical variables by computing their frequencies and percentages, and the quantitative variables will be computed by their mean $\pm \mathrm{SD} /$ median (IQR), as appropriate. Mean scores will be reported for resilience and QoL. The multiple 
linear regression technique will be used to evaluate the effect of independent variables on the outcomes-resilience and QoL for head and neck patients and brain tumour patients. Adjusted $\beta$-coefficients with $95 \% \mathrm{CI}$ will be reported. A p value of $<0.05$ will be considered statistically significant. To assess the relationship between resilience and QoL, correlation analysis will also be performed, using the Pearson or Spearman rank correlation coefficients as appropriate.

\section{Ethical considerations}

Participants will be recruited from the surgical/oncology clinics of AKUH. Written informed consent will be taken from the participants by trained research assistants, after explaining the study procedure and its potential risks and benefits to them.

In this particular study, the participants might feel anxious/uncomfortable during the interview, especially when their stress and depression level will be evaluated. To overcome this, proper training will be given to the research team for sensitive questions. On spot counselling by a trained psychologist will be provided to the participants identified as having depression. Those patients identified with severe depression especially with suicidal intentions will be referred to a psychiatrist.

Strict confidentiality and privacy rules will be maintained and the participants' information will be kept confidential. Interviews will be conducted in a separate room. All study materials containing personal identifiers will be kept in a locked file cabinet. A unique study identification number will be assigned to each participant. Data will be entered in a password-protected electronic database that will only be accessible by the research team.

\section{Patient and public involvement}

This will be a cross-sectional study design and the participants will be interviewed, regarding their sociodemographic factors, anxiety, depression, resilience and QoL, by trained research assistants.

The study findings will be disseminated to different stakeholders, such as healthcare professionals, rehabilitation experts, psychologists and cancer patients through: publications at local, national and international journals, presentations at conferences and workshops and through research briefs.

Acknowledgements We would like to acknowledge our secretarial support, $\mathrm{Mr}$ Mirza Anas. We are also grateful to Dr Wajeeha Zahid for her valuable support. We would also like to acknowledge EORTC (European Organization of Research and Treatment of Cancer) who gave us permission to use their quality of life tool for this study.

Contributors NZ conceived the study, wrote and critically reviewed the manuscript. WK directly overlooked all aspects of study, wrote and critically reviewed the manuscript. SSB and KA intellectually contributed to the study. IA, AAJ and NA reviewed the study for overall quality and design robustness. MK and AE assisted as experts and informed aspects of development of the study intellectually. All authors have contributed intellectually to this manuscript. All authors have read and approved the final manuscript.

Funding The study is funded by Aga khan university seed money funds grant number PF98/0417.
Competing interests None declared.

Patient consent for publication Not required.

Ethics approval Study protocol is approved by Aga khan university ethical review committee with ERC \# 5154-Sur-ERC-17.

Provenance and peer review Not commissioned; externally peer reviewed.

Data availability statement There are no data in this work. No data are available.

Open access This is an open access article distributed in accordance with the Creative Commons Attribution Non Commercial (CC BY-NC 4.0) license, which permits others to distribute, remix, adapt, build upon this work non-commercially, and license their derivative works on different terms, provided the original work is properly cited, appropriate credit is given, any changes made indicated, and the use is non-commercial. See: http://creativecommons.org/licenses/by-nc/4.0/.

\section{REFERENCES}

1. WHO. Cancer fact sheet 2018. Available: http://wwwwhoint/ mediacentre/factsheets/fs297/en/;Lat [Accessed 16 Mar 2018].

2. Parkin DM, Bray F, Ferlay J, et al. Global cancer statistics, 2002. CA Cancer J Clin 2005;55:74-108.

3. Curado MP, Hashibe M. Recent changes in the epidemiology of head and neck cancer. Curr Opin Oncol 2009;21:194-200.

4. Henriksen L, Feighery EC, Schleicher NC, et al. Is adolescent smoking related to the density and proximity of tobacco outlets and retail cigarette advertising near schools? Prev Med 2008;47:210-4.

5. Yusuf A. Cancer care in Pakistan. Jpn J Clin Oncol 2013;43:771-5.

6. Leung SW, Lee T-F, Chien C-Y, et al. Health-related quality of life in 640 head and neck cancer survivors after radiotherapy using EORTC QLQ-C30 and QLQ-H\&N35 questionnaires. BMC cancer 2011;11:128.

7. Bottomley A. The cancer patient and quality of life. Oncologist 2002;7:120-5.

8. Singer S, Das-Munshi J, Brähler E. Prevalence of mental health conditions in cancer patients in acute care--a meta-analysis. Ann Oncol 2010;21:925-30.

9. Kroenke K, Theobald D, Wu J, et al. The association of depression and pain with health-related quality of life, disability, and health care use in cancer patients. J Pain Symptom Manage 2010;40:327-41.

10. Weisman AD. A model for psychosocial phasing in cancer. Gen Hosp Psychiatry 1979;1:187-95.

11. Lawford J, Eiser C. Exploring links between the concepts of quality of life and resilience. Pediatr Rehabil 2001;4:209-16.

12. Epping-Jordan JE, Compas BE, Osowiecki DM, et al. Psychological adjustment in breast cancer: processes of emotional distress. Health Psychol 1999;18:315-26.

13. Richardson GE. The metatheory of resilience and resiliency. J Clin Psychol 2002;58:307-21.

14. Arillo-Santillan E, Lazcano-Ponce E, Hernandez-Avila M, et al. Associations between individual and contextual factors and smoking in 13,293 Mexican students. Am J Prev Med 2005;28:41-51.

15. Resnick B, Galik E, Dorsey S, et al. Reliability and validity testing of the physical resilience measure. Gerontologist 2011;51:643-52.

16. Ryan L, Caltabiano ML. Development of a new resilience scale: the resilience in midlife scale (rim scale). Asian Social Science 2009;5.

17. Wilks SE. Psychometric evaluation of the shortened resilience scale among Alzheimer's caregivers. Am J Alzheimers Dis Other Demen 2008;23:143-9.

18. Abrams MS. Resilience in ambiguous loss. Am J Psychother 2001;55:283-91.

19. Leplège $A$, Hunt $S$. The problem of quality of life in medicine. JAMA 1997;278:47-50.

20. Brix C, Schleussner C, Füller J, et al. The need for psychosocial support and its determinants in a sample of patients undergoing radiooncological treatment of cancer. J Psychosom Res 2008;65:541-8.

21. Strauss B, Brix C, Fischer S, et al. The influence of resilience on fatigue in cancer patients undergoing radiation therapy (RT). $J$ Cancer Res Clin Oncol 2007;133:511-8.

22. Wu L-M, Sheen J-M, Shu H-L, et al. Predictors of anxiety and resilience in adolescents undergoing cancer treatment. J Adv Nurs 2013;69:158-66.

23. Ragnarsdóttir LD. Resilience in men with prostate cancer: relationship between resilience, social support and distress 2012.

24. Orom H, Nelson CJ, Underwood W, et al. Factors associated with emotional distress in newly diagnosed prostate cancer patients. Psychooncology 2015;24:1416-22. 
25. Min J-A, Yoon S, Lee C-U, et al. Psychological resilience contributes to low emotional distress in cancer patients. Supportive Care in Cancer 2013;21:2469-76.

26. Tian J, Hong J-S. Assessment of the relationship between resilience and quality of life in patients with digestive cancer. World $\mathrm{J}$ Gastroenterol 2014;20.

27. Dunne S, Mooney O, Coffey L, et al. Psychological variables associated with quality of life following primary treatment for head and neck cancer: a systematic review of the literature from 2004 to 2015. Psychooncology 2017;26:149-60.

28. W-W W, Tsai S-Y, Liang S-Y, et al. The mediating role of resilience on quality of life and cancer symptom distress in adolescent patients with cancer. Journal of Pediatric Oncology Nursing 2015;1043454214563758.

29. Melo Filho MRde, Rocha BA, Pires MBdeO, et al. Quality of life of patients with head and neck cancer. Braz J Otorhinolaryngol 2013;79:82-8.

30. Talepasand S, Pooragha F, Kazemi M. Resiliency and quality of life in patients with cancer: moderating role of duration of awareness of cancer. Iran J Cancer Prev 2013;6:222-6.

31. Rehman B, ud Din Q, Khan M, et al. Evaluation of the quality of life in patients with oral squamous cell carcinoma. Cell 2012;333:9199288.

32. Mahalakshmi P, Vanisree A. Quality of life measures in glioma patients with different grades: a preliminary study. Indian journal of cancer 2015;52:580.

33. Chiu L, Chiu N, Zeng L, et al. Quality of life in patients with primary and metastatic brain cancer as reported in the literature using the EORTC QLQ-BN20 and QLQ-C30. Expert Rev Pharmacoecon Outcomes Res 2012;12:831-7.

34. Kim C-W, Joo J-D, Kim Y-H, et al. Health-Related quality of life in brain tumor patients treated with surgery: preliminary result of a single institution. Brain Tumor Res Treat 2016;4:87-93.

35. Okita $Y$, Narita $Y$, Miyahara R, et al. Health-Related quality of life in long-term survivors with grade II gliomas: the contribution of disease recurrence and Karnofsky performance status. Jpn J Clin Oncol 2015;45:hyv115-913.

36. Dowrick C, Kokanovic R, Hegarty K, et al. Resilience and depression perspectives from primary care. Health 2008;12:439-52.

37. Davydov DM, Stewart R, Ritchie K, et al. Resilience and mental health. Clin Psychol Rev 2010;30:479-95

38. Wagnild GM, Guinn PE. The resilience scale user's guide: for the US English version of the resilience scale TM and the 14-Item resilience scale TM (RS-14 TM). Resilience center, 2009.

39. Bhamani SS, Pasha O, Karmaliani R, et al. Validation of the Urdu Version of Wagnild and Young's Long and Short Resilience Scales Among 20- to 40-Year-Old Married Women Living in Urban Squatter Settlements of Karachi, Pakistan. J Nurs Meas 2015;23:425-35.

40. Abubakar S, Isezuo S. Health related quality of life of stroke survivors: experience of a stroke unit. Int J Biomed Sci 2012;8:183-7.

41. Fayers PM, Aaronson NK, Bjordal K, et al. Eortc QLQ-C30 scoring manual, 2001.

42. Polit DF, Beck CT. The content validity index: are you sure you know what's being reported? critique and recommendations. Res Nurs Health 2006;29:489-97.

43. Sangoseni O, Hellman M, Hill C. Development and validation of a questionnaire to assess the effect of online learning on behaviors, attitudes, and clinical practices of physical therapists in the United States regarding evidence-based clinical practice. IJAHSP 2013;11.

44. Zigmond AS, Snaith RP. The hospital anxiety and depression scale. Acta Psychiatr Scand 1983;67:361-70.

45. Mumford DB, Tareen IAK, Bajwa MAZ, et al. The translation and evaluation of an Urdu version of the hospital anxiety and depression scale. Acta Psychiatr Scand 1991;83:81-5.

46. Khalid W, Rozi S, Ali TS, et al. Quality of life after stroke in Pakistan. BMC Neurol 2016;16:250.

47. Haase JE. The adolescent resilience model as a guide to interventions. J Pediatr Oncol Nurs 2004;21:289-99. 